\title{
Giant congenital left main coronary artery fistula to the superior vena cava in an adult patient with coronary artery disease
}

\author{
Ilias A. Kouerinis, MD, PhD, ${ }^{a}$ Martha Deligianni, MD, ${ }^{\mathrm{b}}$ Vassilios Maniatis, MD, PhD, ${ }^{\mathrm{b}}$ \\ Ilias Elefteriadis, MD, ${ }^{\mathrm{b}}$ Antonios Kourtesis, MD, ${ }^{\mathrm{a}}$ and Nikolaos Michalopoulos, MD, \\ Athens, Greece
}

丹 Supplemental material is available online.

I $\mathrm{t}$ is well established that coronary artery fistulae (CAFs) can cause dyspnea, fatigue, angina (7\%), and infarction (3\%) as a result of the coronary steal phenomenon. ${ }^{1}$ Nevertheless, there are few reports in which a CAF was associated with coronary artery disease (CAD), and even fewer studies have examined the consequences of this association on coronary hemodynamics and myocardial blood flow. ${ }^{2,3}$

We present the surgical treatment of a giant left main coronary artery (LMCA) fistula draining into the superior vena cava and coexistent $\mathrm{CAD}$, and we investigate the contribution of the coronary steal syndrome to this patient's symptoms.

\section{Clinical Summary}

A 62-year-old man was referred to our department with the diagnosis of an LMCA fistula and double-vessel disease (left anterior descending coronary artery, 85\%; circumflex artery, 80\%; Figures 1, E1, and E2). Oxymetry had shown an increased oxygen saturation of $84 \%$ at the lower segment of the superior vena cava with high right ventricular filling pressures, and the Qp/Qs ratio was calculated at $1.4 / 1$.

Intraoperatively, after the opening of the pericardium, the fistula was easily identified (Figure 2), and cardiopulmonary bypass was initiated. After clamping the aorta, we provided retrograde cardioplegia to maintain arrest because significant shunt was noticed during the antegrade distribution. This problem was subsequently solved with digital pressure on the fistula, which was finally ligated $3 \mathrm{~cm}$ distally to the LMCA. We considered that it was too dangerous to attempt to ligate it more proximally to the LMCA because extreme calcification was palpated there. The check for step-up in oxygen saturation at the level of the right

From the Department of Cardiac Surgery, Aghia Sophia Children's Hospital, ${ }^{\mathrm{a}}$ Athens, Greece; and Iaso General Hospital, ${ }^{\mathrm{b}}$ Athens, Greece.

Received for publication June 12, 2007; accepted for publication July 5, 2007.

Address for reprints: Ilias Kouerinis, MD, PhD, 46 Doukissis Plakentias, Melissia, Athens, Greece (E-mail: ikouerinis@hotmail.com).

J Thorac Cardiovasc Surg 2007;134:1057-8

$0022-5223 / \$ 32.00$

Copyright () 2007 by The American Association for Thoracic Surgery doi:10.1016/j.jtcvs.2007.07.004 atrium and inferior vena cava proved negative, and we continued the operation by bypassing the first obtuse marginal and left

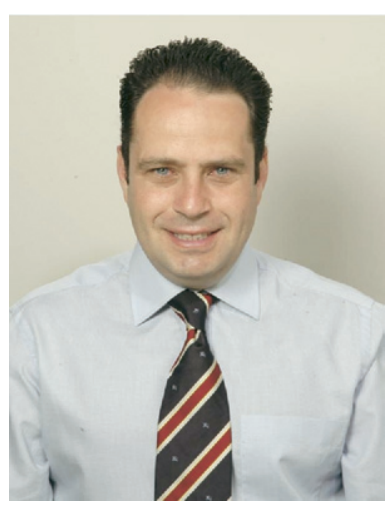

Dr Kouerinis anterior descending coronary artery with a saphenous vein graft and the left internal thoracic artery consecutively. The postoperative course was uneventful, and the patient was discharged home in good condition.

\section{Discussion}

Although only $40 \%$ of patients aged older than 20 years with CAF are asymptomatic, ${ }^{4}$ the exact role of CAFs as a nonatherosclerotic cause of myocardial ischemia has not been fully studied. Stierle and colleagues, ${ }^{5}$ using a sinus lactate study, demonstrated the role of the steal mechanism of CAFs, whereas Bamoshmoosh and associates $^{2}$ studied the reverse phenomenon, the redistribution of blood flow from the fistula to the coronary vessel. This so-called reverse steal phenomenon might offer an explanation as to how patients with severe $\mathrm{CAD}$ and remarkable shunt resulting from a CAF can survive so long.

Nevertheless, it still remains difficult to explain how a Qp/Qs ratio of 1.4 or more resulting exclusively from a big LMCA fistula is compatible with life, when we all know that the overall coronary blood flow in rest represents only $4 \%$ of systemic circulation.

From a pathophysiologic point of view, we would propose the categorization of CAFs as extramyocardial (ie, fistulae with a drainage site different from a cardiac ventricle) and intramyocardial (ie, fistulae with a drainage point into a heart cavity). We estimate that in any case of extramyocardial CAF, the main volume of blood flow encountered as left-to-right shunt is shunting during the systolic period of the cardiac cycle, when perfusion of the myocardium is minimal. This assessment is confirmed from an angiogram of our patient in which the fistula is mainly demonstrated during systole and before the coronary arteries (Figure E3). Similarly, we can assume that during diastole the shunt is much less, or the reverse steal phenomenon might also take place (ie, the squeezed coronary artery, when decompressed during diastole, pumps blood from the fistula), delaying in this way the onset of symptoms until the occluding lesion becomes more critical.

Obviously in cases of intramyocardial CAFs (eg, from a coronary artery to the right ventricle [40\% of cases]), the pathophysiologic mechanism is much different. During systole, the shunt from the fistula to the heart cavity is minimal because the fistula is compressed through its myocardial course. During diastole, the distribution of blood from the supplying coronary artery to the fistula and the distal coronary vessel is basically dependent on the vascular resistances of these competitive vessels. The total shunt of 


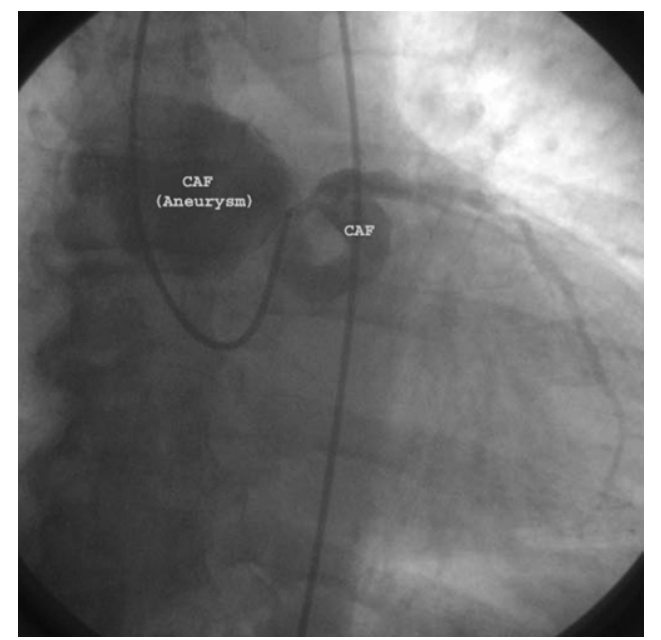

Figure 1. Coronary angiogram analysis demonstrating the fistula. Note that the catheter has just inserted into the LMCA ostium. $C A F$, Coronary artery fistula; $L M C A$, left main coronary artery.

intramyocardial fistulae should be much less when compared with that of extramyocardial fistulae with similar dimensions. According to all the above, CAFs draining into the right atrium (25\% of cases) might show a physiology more similar to that of extramyocardial CAFs.

Concerning the association of CAFs with CAD, only few reports are available. ${ }^{2}$ In such cases the position of the coronary stenosis in relation to the CAF is of main importance. It seems that when the coronary stenosis is before the CAF, it reduces the hemodynamic effect of the CAF. The condition becomes more critical when the coronary stenosis is below the CAF, as in our patient; in this case, the combination of CAD and CAF may contribute to inducement of myocardial ischemia. Two cases of atherosclerosis development with significant stenosis at the origin of the fistula have been reported as well.

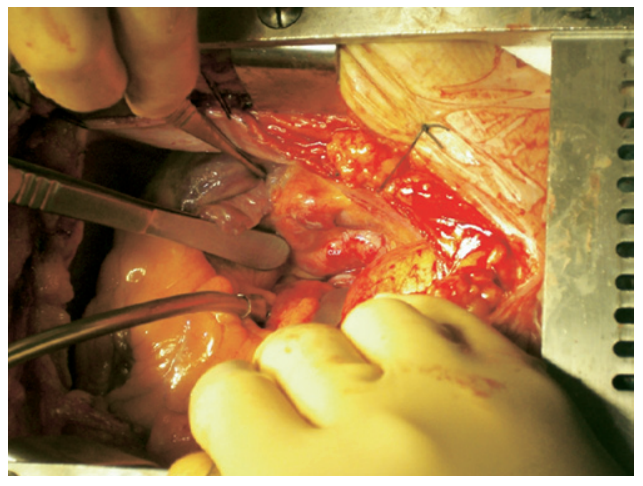

Figure 2. Our intraoperative findings.

\section{Conclusions}

The exact contribution of a CAF to the symptoms of a patient with coexistent $\mathrm{CAD}$ is difficult to evaluate. Extramyocardial fistulae originating proximal to an occluding lesion are the most prominent in their contribution to the early onset of symptoms in a patient with CAD.

\section{References}

1. Iadanza A, Del Pasqua A, Fineschi M, et al. Three-vessel left ventricular microfistulization syndrome. A rare case of angina. Int $J$ Cardiol. 2004;96:109-11.

2. Bamoshmoosh M, Marraccini P, Pratali L, et al. "Reverse steal phenomenon" in a patient with coronary artery disease and coronary-left ventricular fistula. Int J Cardiol. 2007;115:e33-5.

3. Papazoglou PD, Mitsibounas D, Nanas JN. Left anterior descending coronary artery-left ventricular fistula presenting as unstable angina and syncope. Int J Cardiol. 2004;96:121-2.

4. Makaryus A, Stechel R, Green S. A rare combination of left main coronary fistula to left superior vena cava with drainage into the coronary sinus in a 74-year-old woman. J Invasive Cardiol. 2003;15: 270-2.

5. Stierle U, Gianitisis E, Sheikhzadeh A, et al. Myocardial ischemia in generalized coronary artery-left ventricular microfistulae. Int J Cardiol. $1998 ; 63: 47-52$ 


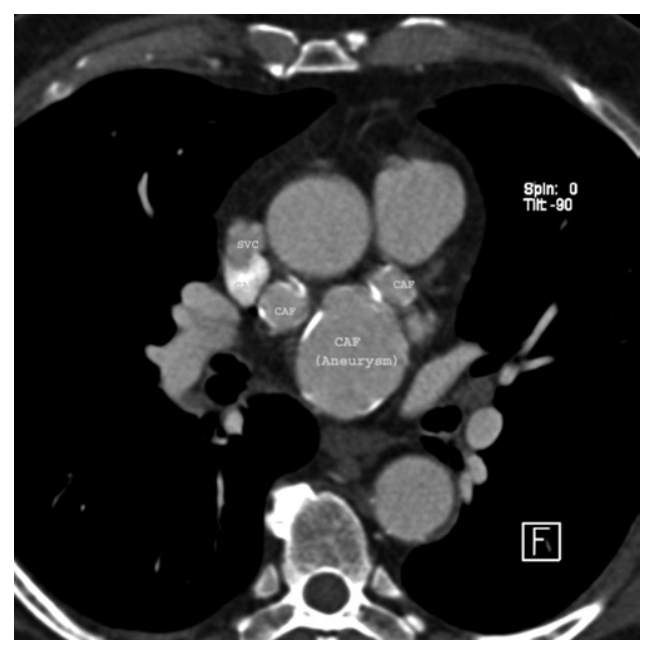

Figure E1. Axial computed tomographic scan (after intravenous contrast) at the level of the aortopulmonary window. SVC, Superior vena cava; CAF, coronary artery fistula.

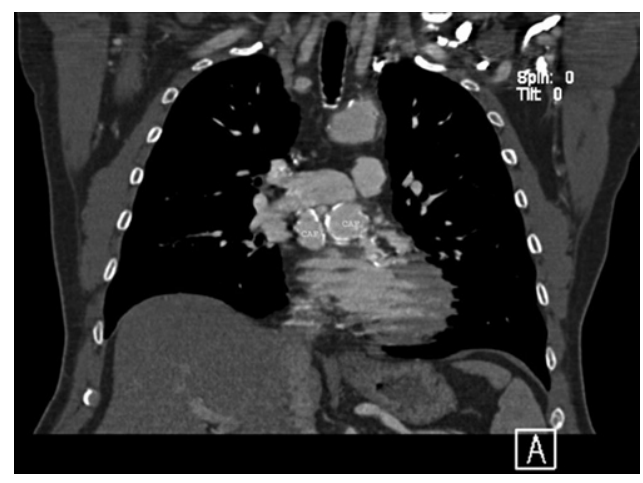

Figure E2. Coronal computed tomographic scan (after intravenous contrast) at the level of the aortopulmonary window. CAF, Coronary artery fistula. 


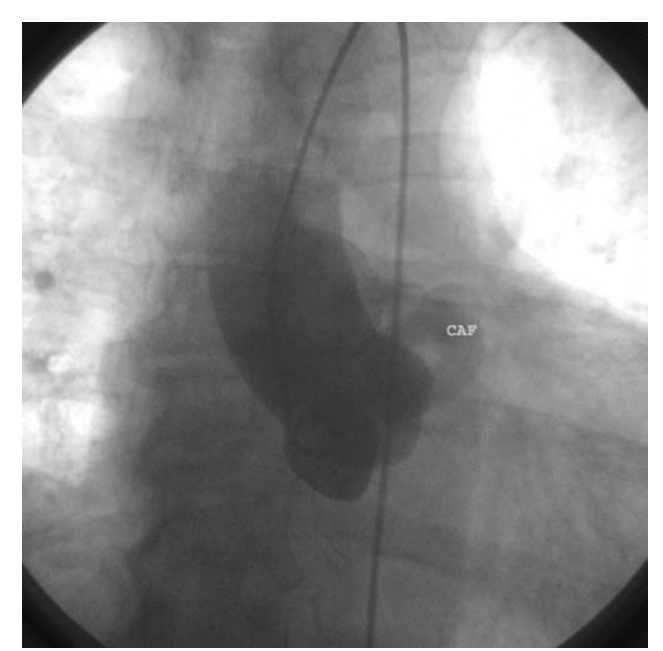

Figure E3. Careful examination with a low-speed coronary angiograph revealed that the coronary artery fistula (CAF) was mainly demonstrated during the systolic period and before the opacification of the coronary arteries. 